\title{
JACOBIANS OF SOBOLEV HOMEOMORPHISMS
}

\author{
STANISLAV HENCL AND JAN MALÝ
}

\begin{abstract}
Let $\Omega \subset \mathbb{R}^{n}$ be a domain. We show that each homeomorphism $f$ in the Sobolev space $W_{\text {loc }}^{1,1}\left(\Omega, \mathbb{R}^{n}\right)$ satisfies either $J_{f} \geq 0$ a.e or $J_{f} \leq 0$ a.e. if $n=2$ or $n=3$. For $n>3$ we prove the same conclusion under stronger assumption that $f \in W_{\text {loc }}^{1, s}\left(\Omega, \mathbb{R}^{n}\right)$ for some $s>[n / 2]$ (or in the setting of Lorentz spaces).
\end{abstract}

\section{INTRODUCTION}

In this paper we address the following issue. Suppose that $\Omega \subset \mathbb{R}^{n}$ is a domain and that $f: \Omega \rightarrow \mathbb{R}^{n}$ is a homeomorphism of the Sobolev class $W_{\text {loc }}^{1, s}\left(\Omega ; \mathbb{R}^{n}\right), s \geq 1$. Here $W_{\text {loc }}^{1, s}\left(\Omega ; \mathbb{R}^{n}\right)$ consists of all locally $s$-integrable mappings of $\Omega$ into $\mathbb{R}^{n}$ whose coordinate functions have locally $s$-integrable distributional derivatives. Is it true that the jacobian $J_{f}$ (the determinant of the matrix of derivatives) is either non-negative almost everywhere or nonpositive almost everywhere? It is well-known that every homeomorphism on the domain is either sense-preserving or sense-reversing (see Preliminaries for the definition) and therefore we can ask whether each sense-preserving homeomorphism in the Sobolev space $W_{\text {loc }}^{1, s}$ satisfies $J_{f} \geq 0$ almost everywhere. Roughly speaking, we are interested in the question whether topological and analytical definitions of orientation lead to the same result.

Our research is motivated by our interest in geometric function theory, where the nonnegativity of the jacobian is a standing assumption. We would like to know that this is not an essential restriction at least for homeomorphisms. Note that recent developments in geometric function theory find new inspiration in nonlinear elasticity and other problems of calculus of variations. For an overwiew of the field, discussion of interdisciplinary links and further references see [7].

The problem is mostly formulated as a question whether the Jacobian of a Sobolev homeomorphism can change the sign on a set of positive measure. We know it from Hajłasz [5], who constructed (not yet published) examples of a.e. approximatively differentiable homeomorphisms with Jacobian of alternating sign. However, their gradient is not integrable, so they are not Sobolev mappings. The problem has been also explicitly formulated in [11]. Let us mention some further difficulties we should keep in mind. There exists a Sobolev (even Lipschitz) homeomorphism with Jacobian vanishing on a set of positive measure (see [8] or [7], Subsection 6.5.6). For $1 \leq s<n$,

2000 Mathematics Subject Classification. 26B10, 46E35.

Key words and phrases. Sobolev mapping, homeomorphism, Jacobian, orientation.

The first author was supported by the research project MSM 0021620839 of the Czech Ministry MŠMT. The second author was supported in part by the MSM 0021620839 and in part by the grant GA ČR 201/09/0067. 
there exists a mapping $f \in W^{1, s}\left(B(0,1) ; \mathbb{R}^{n}\right) \cap C\left(\bar{B}(0,1) ; \mathbb{R}^{n}\right)$ such that $f$ coincides with the identity on $\partial B(0,1)$ but $J_{f}<0$ a.e., see [12]. This mapping, however, is not a homeomorphism.

A full correspondence of topological and analytical definitions of orientation would mean simultaneous validity of the following assertions:

Conjecture A. Let $\Omega \subset \mathbb{R}^{n}$ be an open set and $f \in W_{\text {loc }}^{1,1}\left(\Omega, \mathbb{R}^{n}\right)$ is a sense preserving homeomorphism. Then $J_{f} \geq 0$ a.e.

Conjecture B. Let $\Omega \subset \mathbb{R}^{n}$ be an open set and $f \in W_{\text {loc }}^{1,1}\left(\Omega, \mathbb{R}^{n}\right)$ is a homeomorphism. If $J_{f} \geq 0$ a.e., then $f$ is sense preserving.

If Conjecture A is true, then an eventual counterexample to Conjecture B should satisfy $J_{f}=0$ a.e.

It is clear that homeomorphism of finite distortion (see [7] for the definition and basic properties) cannot satisfy $J_{f}=0$ a.e., but on the other hand it is not difficult to find a homeomorphism in $B V$ such that $J_{f}=0$ a.e. This has the form $f\left(x_{1}, x_{2} \ldots, x_{n}\right)=\left(\varphi\left(x_{1}\right), x_{2}, \ldots, x_{n}\right)$ where $\varphi$ is a one-dimensional example based on iteration of the standard Cantor-Vitali one-third function.

We focus our attention to the statement of Conjecture A. Our Theorem 1.1 gives an affirmative solution of our problem in low dimension, particularly in the "physically relevant" dimension $n=3$, without any restriction.

Theorem 1.1. Let $\Omega \subset \mathbb{R}^{n}$ be an open set and $n \leq 3$. Suppose that $f \in$ $W_{\mathrm{loc}}^{1,1}\left(\Omega, \mathbb{R}^{n}\right)$ is a sense preserving homeomorphism. Then $J_{f} \geq 0$ a.e.

This is obvious in dimension $n=1$ since each homeomorphism is either increasing or decreasing function and therefore satisfies $f^{\prime} \geq 0$ a.e. or $f^{\prime} \leq 0$ a.e. Also in the planar case $n=2$, the statement of Theorem 1.1 was known and actually follows pretty easily from the result of Gehring and Lehto [4]. See also [1], Section 3.3., where the statement is also generalized to open and discrete Sobolev mappings. To keep the presentation more self-contained we will prove the two-dimensional version of Theorem 1.1 in Section 3. In fact, we even show the result for any homeomorphism of bounded variation $f \in B V\left(\Omega, \mathbb{R}^{2}\right)$.

The most interesting case $n=3$ is realized in Section 5 . It can be also obtained as a particular case of our general $n$-dimensional result, but we treat it separately to demonstrate the idea in the simplest form.

Before stating what we are able to prove in the general $n$-dimensional case, we note that for the sake of sharpness we use the fine scale of Lorentz spaces. For the definition of the space $L_{p, 1}(\Omega)$ in consideration we refer to the beginning of Section 6 . Recall that

$$
L^{p}(\Omega) \subset L_{p, 1}(\Omega) \subset \bigcap_{s>p} L^{s}(\Omega) .
$$

As we state below, we are able to prove the desired property for mappings with gradient in $L_{p, 1}$ with $p=[n / 2]$, which means the integer part of $n / 2$. (This is, $[n / 2]=1,1,2,2, \ldots$ for $n=2,3,4,5, \ldots$ ) Thus, within the crude scale of ordinary Sobolev spaces, the result holds for $W^{1, s}$-mappings with $s>p$. 
Theorem 1.2. Let $\Omega \subset \mathbb{R}^{n}$ be an open set, $n \geq 2$. Suppose that $f: \Omega \rightarrow \mathbb{R}^{n}$ is a sense preserving Sobolev homeomorphism with $\nabla f \in L_{p, 1}$, where $p=$ $[n / 2]$. Then $J_{f} \geq 0$ a.e.

Let us compare our results with previously known facts. The conjecture was known for homeomorphisms in $W^{1, s}, s>n-1$, (see [3, Lemma 5.10, Theorem 5.21]) and [17] for a generalization to discontinuous functions). Although the result has been supposed to be understood for $s=n-1$ [5], we are not aware of any explicit statement in the literature. The argument for $s>n-1$ is based on Sobolev embedding theorem on spheres and therefore it does not work for $s<n-1$. To obtain the results in our setting we have to include new ideas and techniques. The main new tool we use is the theory of linking numbers and its topological invariance (see Section 4 for details). Note that another application of linking spheres in Sobolev function theory recently occured in [18].

\section{Preliminaries}

We define $\mathbb{B}_{k}(r)$ as the ball with center at 0 and radius $r$ in $\mathbb{R}^{k}$. The sphere $\mathbb{S}_{k-1}(r)$ is the boundary of $\mathbb{B}_{k}(r)$. We omit to mark the parameter $r$ if $r=1$. We denote by $\left(\mathbf{e}_{1}, \ldots, \mathbf{e}_{k}\right)$ the canonical basis of $\mathbb{R}^{k}$, the dimension $k$ is to be deduced from the context.

It is known that functions in the Sobolev space $f \in W_{\text {loc }}^{1,1}$ are approximatively differentiable almost everywhere and moreover that

$$
\lim _{r \rightarrow 0+} f_{B\left(x_{0}, r\right)}\left|\frac{f(x)-f\left(x_{0}\right)-\nabla f\left(x_{0}\right)\left(x-x_{0}\right)}{r}\right| d x=0
$$

for almost every $x_{0}$. This follows easily from e.g. [2, 6.1.2, Theorem 2].

Topological degree. Given a smooth map $f$ from $\Omega \subset \mathbb{R}^{n}$ into $\mathbb{R}^{n}$ we can define the topological degree as

$$
\operatorname{deg}\left(f, \Omega, y_{0}\right)=\sum_{\{x \in \Omega: f(x)=y\}} \operatorname{sgn}\left(J_{f}(x)\right)
$$

if $J_{f}(x) \neq 0$ for each $x \in f^{-1}(y)$. This definition can be extended to arbitrary continuous mappings and each point see e.g. [3].

A continuous mapping $f: \Omega \rightarrow \mathbb{R}^{n}$ is called sense-preserving if

$$
\operatorname{deg}\left(f, \Omega^{\prime}, y_{0}\right)>0
$$

for all domains $\Omega^{\prime} \subset \subset \Omega$ and all $y_{0} \in f\left(\Omega^{\prime}\right) \backslash f\left(\partial \Omega^{\prime}\right)$. Similarly we call $f$ sense-reversing if $\operatorname{deg}\left(f, \Omega^{\prime}, y_{0}\right)<0$ for all $\Omega^{\prime}$ and $y_{0}$. Let us recall that each homeomorphism on a domain is either sense-preserving or sense-reversing see [13, II.2.4., Theorem 3].

We will use the fact that the topological degree is stable under homotopy. That is for every continuous mapping $H: \bar{\Omega} \times[0,1] \rightarrow \mathbb{R}^{n}$ and $p \in \mathbb{R}^{n}$ such that $p \notin H(\partial \Omega, t)$ for all $t \in[0,1]$ we have

$$
\operatorname{deg}(H(\cdot, 0), \Omega, p)=\operatorname{deg}(H(\cdot, 1), \Omega, p) .
$$




\section{Planar case}

Proof of Theorem 1.1 for $n=2$. It is well-known fact that each $W^{1,1}$ function is absolutely continuous on almost all lines parallel to coordinate axes (see e.g. [14, I.1.2]) and therefore it has classical partial derivatives almost everywhere. By Gehring and Lehto Theorem [4] it follows that $f$ is differentiable almost everywhere.

Let us consider a point $x_{0} \in \Omega$ such that $f$ is differentiable at $x_{0}$ and $J_{f}\left(x_{0}\right) \neq 0$. Then $\alpha=\inf _{\|y\|=1}\left|D f\left(x_{0}\right) y\right|>0$ and we can choose $r>0$ small enough such that

$$
\left|f\left(x_{0}+x\right)-f\left(x_{0}\right)-D f\left(x_{0}\right) x\right|<r \alpha \text { for every } x \in \mathbb{S}_{1}(r) .
$$

Let us consider a homotopy

$$
H(x, t)=(1-t)\left(f\left(x_{0}+x\right)-f\left(x_{0}\right)\right)+t D f\left(x_{0}\right) x \text { for } x \in \mathbb{B}_{2}(r) .
$$

By (3.1) we obtain that $0 \notin H\left(\mathbb{S}_{n-1}(r), t\right)$ for every $t \in[0,1]$ and thus

$$
\begin{aligned}
\operatorname{deg}\left(f(x), x_{0}+\mathbb{B}_{2}(r), f\left(x_{0}\right)\right) & =\operatorname{deg}\left(f\left(x_{0}+x\right)-f\left(x_{0}\right), \mathbb{B}_{2}(r), 0\right)= \\
& =\operatorname{deg}\left(D f\left(x_{0}\right) x, \mathbb{B}_{2}(r), 0\right)=\operatorname{sgn} J_{f}\left(x_{0}\right) .
\end{aligned}
$$

Remark 3.1. It is known that any $B V$ homeomorphism in the plane is also differentiable almost everywhere (see e.g. [6]). Therefore, analogously as above we obtain that any sense-preserving homeomorphism in $B V\left(\Omega, \mathbb{R}^{2}\right)$ satisfies $J_{f} \geq 0$ a.e. Here $J_{f}$ denotes the determinant of the absolutely continuous part of $D f$. We expect that the conjecture is true for any $B V$ homeomorphisms in arbitrary dimension.

\section{LINKING NUMBER}

Let $n, p, q$ be positive integers with $p+q=n-1$. Let us consider the mapping $\Phi(\xi, \eta): \overline{\mathbb{B}}_{p+1} \times \overline{\mathbb{B}}_{q+1} \rightarrow \mathbb{R}^{n}$ defined coordinatewise as $\Phi(\xi, \eta)=x$, where

$$
\begin{aligned}
x_{1} & =\left(2+\eta_{1}\right) \xi_{1}, \\
& \cdots \\
x_{p+1} & =\left(2+\eta_{1}\right) \xi_{p+1}, \\
x_{p+2} & =\eta_{2}, \\
& \ldots \\
x_{p+q+1} & =\eta_{q+1} .
\end{aligned}
$$

Denote by $\mathbb{A}$ the anuloid

$\Phi\left(\mathbb{S}_{p} \times \mathbb{B}_{q+1}\right)=\left\{x \in \mathbb{R}^{n}:\left(\sqrt{x_{1}^{2}+\ldots+x_{p+1}^{2}}-2\right)^{2}+x_{p+2}^{2}+\ldots+x_{n}^{2}<1\right\}$.

Of course given $x \in \overline{\mathbb{A}}$ we can find a unique $\xi \in \mathbb{S}_{p}$ and $\eta \in \overline{\mathbb{B}}_{q+1}$ such that $\Phi(\xi, \eta)=x$. We will denote these as $\xi(x)$ and $\eta(x)$.

A link is a pair $(\varphi, \psi)$ of parametrized surfaces $\varphi: \mathbb{S}_{p} \rightarrow \mathbb{R}^{n}, \psi: \mathbb{S}_{q} \rightarrow \mathbb{R}^{n}$. The linking number of the link $(\varphi, \psi)$ is defined as the topological degree

$$
£(\varphi, \psi)=\operatorname{deg}(L, \mathbb{A}, 0),
$$


where the mapping $L=L_{\varphi, \psi}: \overline{\mathbb{A}} \rightarrow \mathbb{R}^{n}$ is defined as

$$
\begin{gathered}
L(x)=\varphi(\xi(x))-\bar{\psi}(-\eta(x)) \text { or equivalently } \\
L(\Phi(\xi, \eta))=\varphi(\xi)-\bar{\psi}(-\eta), \quad \xi \in \mathbb{S}_{p}, \eta \in \mathbb{B}_{q+1},
\end{gathered}
$$

where $\bar{\psi}$ is an arbitrary continuous extension of $\psi$ to $\overline{\mathbb{B}}_{q+1}$ (of course, the degree does not depend on the way how we extend $\psi$, it depends only on the values on the boundary $\partial \mathbb{A}=\Phi\left(\mathbb{S}_{p} \times \mathbb{S}_{q}\right)$ ). Geometrically speaking, for $p=q=1$, the linking number is the number of loops of a curve $\varphi$ around a curve $\psi$ counting orientation into account as +1 or -1 . For the introductions to the linking number in $\mathbb{R}^{3}$ and its application to the theory of knots see [15].

The canonical link is the pair $(\mu, \nu)$, where

$$
\begin{aligned}
& \mu(\xi)=\Phi(\xi, 0), \quad \xi \in \mathbb{S}_{p}, \\
& \nu(\eta)=\Phi\left(\mathbf{e}_{1}, \eta\right), \quad \eta \in \mathbb{S}_{q} .
\end{aligned}
$$

For example in dimension $n=3$ we get that

$$
\begin{aligned}
& \mu\left(\mathbb{S}_{1}\right)=\left\{x \in \mathbb{R}^{3}: x_{3}=0, x_{1}^{2}+x_{2}^{2}=4\right\} \text { and } \\
& \nu\left(\mathbb{S}_{1}\right)=\left\{x_{2}=0,\left(x_{1}-2\right)^{2}+x_{3}^{2}=1\right\} .
\end{aligned}
$$

It is well known, however difficult to find, that the linking number is a topological invariant. We will provide a simple proof for the case that we just need, namely for mapping of the canonical link.

Proposition 4.1. Let $n, p, q$ be positive integers with $p+q=n-1$. Let $f: \mathbb{B}_{n}(4) \rightarrow \mathbb{R}^{n}$ be a homeomorphism. Then $£(f \circ \mu, f \circ \nu)$ is 1 if $f$ is sense preserving and -1 if $f$ is sense reversing.

Proof. We consider a homotopy $H(\overline{\mathbb{A}} \times[0,1]) \rightarrow \mathbb{R}^{n}$ defined as

$$
H(\Phi(\xi, \eta), t)=f(\Phi(\xi, t \eta))-f\left(\Phi\left(\mathbf{e}_{1},(t-1) \eta\right)\right), \quad \xi \in \mathbb{S}_{p}, \eta \in \mathbb{B}_{q+1} .
$$

We see that

$$
\begin{aligned}
H(\Phi(\xi, \eta), 0) & =(f \circ \mu)(\xi)-(f \circ \nu)(-\eta), \\
H(x, 1) & =f(x)-f\left(2 \mathbf{e}_{1}\right) .
\end{aligned}
$$

Since $f$ is a homeomorphism, $H(x, t)=0$ for $x=\Phi(\xi, \eta)$ with $\xi \in \mathbb{S}_{p}$ if and only if $\xi=\mathbf{e}_{1}$ and $\eta=0$. This however cannot occur if $x \in \partial \mathbb{A}$. It follows that $£(f \circ \mu, f \circ \nu)=\operatorname{deg}(H(\cdot, 0), \mathbb{A}, 0)$ equals to the $\operatorname{deg}\left(f-f\left(2 \mathbf{e}_{1}\right), \mathbb{A}, 0\right)=$ $\operatorname{deg}\left(f, \mathbb{A}, f\left(2 \mathbf{e}_{1}\right)\right)$ and proves the theorem.

\section{ThreE-Dimensional CASE}

Proof of Theorem 1.1 for $n=3$. Denote $B=B(0,4)$. Let us consider a point $x_{0} \in \Omega$ such that $x_{0}$ is a Lebesgue point of the derivative of $f$, we have (2.1) at $x_{0}$ and $J_{f}\left(x_{0}\right) \neq 0$. Let us consider a sequence $\left\{r_{k}\right\}$ of small radii, $r_{k} \searrow 0$. We may assume that $B\left(x_{0}, 3 r_{k}\right) \subset \Omega$. Next, let $g_{k}$ be the rescaled functions

$$
g_{k}(x)=\frac{f\left(x_{0}+r_{k} x\right)-f\left(x_{0}\right)}{r_{k}},
$$

and $g(x)=\nabla f\left(x_{0}\right) x$. Then a simple change of variables shows that

$$
f_{B}\left|\nabla g_{k}(x)-\nabla g(x)\right| d x=f_{B\left(x_{0}, 4 r_{k}\right)}\left|\nabla f(y)-\nabla f\left(x_{0}\right)\right| d y \stackrel{k \rightarrow \infty}{\rightarrow} 0,
$$


here we use that $x_{0}$ is a Lebesgue point for $\nabla f$. We have verified that $\nabla g_{k} \rightarrow \nabla g$ in $L^{1}\left(B ; \mathbb{R}^{3 \times 3}\right)$. Similarly we observe that

$$
f_{B}\left|g_{k}(x)-g(x)\right| d x=f_{B\left(x_{0}, 4 r_{k}\right)}\left|\frac{f(y)-f\left(x_{0}\right)-\nabla f\left(x_{0}\right)\left(y-x_{0}\right)}{r_{k}}\right| d y \stackrel{k \rightarrow \infty}{\rightarrow} 0
$$

by $(2.1)$ and therefore $g_{k} \rightarrow g$ in $L^{1}\left(B ; \mathbb{R}^{3}\right)$ and hence $g_{k} \rightarrow g$ in $W^{1,1}\left(B ; \mathbb{R}^{3}\right)$. By an appropriate selection of $r_{k}$ we can achieve that $\sum_{k}\left\|g_{k}-g\right\|_{1,1}<\infty$. Given $\eta \in \mathbb{B}_{2}\left(\frac{1}{3}\right)$, we denote

$$
\varphi_{\eta}(\xi)=\Phi(\xi, \eta), \quad \xi \in \mathbb{S}_{1} .
$$

Similarly, given $\xi \in \mathbb{B}_{2}\left(\frac{1}{3}\right)$, we denote

$$
\psi_{\xi}(\eta)=\Phi\left(\mathbf{e}_{1}+\xi, \eta\right), \quad \eta \in \mathbb{S}_{1} .
$$

We say that $T=\varphi_{\eta}\left(\mathbb{S}_{1}\right)$ is a good circle for a function $h \in W^{1,1}(B) \cap$ $C(B)$ if $\left.h\right|_{T} \in W^{1,1}(T)$ and the tangential derivatives of $\left.h\right|_{T}$ coincide with the corresponding directional derivatives of $h$ a.e. with respect to the onedimensional measure on the circle. Let us recall that the restriction of $W^{1,1}$ function to almost every line parallel to coordinate axis is one-dimensional $W^{1,1}$ function (see e.g. [14, Proposition 1.2]) and therefore analogously the restriction to almost every circle is one-dimensional $W^{1,1}$ function.

Since $\Phi$ is a diffeomorphism,

$$
\sum_{k}\left\|g_{k} \circ \Phi-g \circ \Phi\right\|_{W^{1,1}\left(\mathbb{S}_{1} \times \mathbb{B}_{2}\left(\frac{1}{3}\right)\right)}<\infty .
$$

By the Fubini theorem, for almost every $\eta \in \mathbb{B}_{2}\left(\frac{1}{3}\right)$ we obtain that $T=$ $\varphi_{\eta}\left(\mathbb{S}_{1}\right)$ is a good circle for all $g_{k}$ and moreover

$$
\sum_{k} \int_{T}\left(\left|g_{k}(x)-g(x)\right|+\left|\nabla g_{k}(x)-\nabla g(x)\right|\right) d x<\infty
$$

in particular $g_{k} \rightarrow g$ in $W^{1,1}(T)$. It is enough for us just to fix one such a circle $\varphi_{\eta_{0}}$ which we will denote by $\varphi$ from now. Similarly, we find a circle $T^{\prime}=\psi\left(\mathbb{S}_{1}\right)$ with $\psi=\psi_{\xi_{0}}$ such that $g_{k} \rightarrow g$ in $W^{1,1}\left(T^{\prime}\right)$. Using the embedding of one-dimensional Sobolev spaces into the space of continuous functions we infer that $g_{k} \rightarrow g$ uniformly on $T \cup T^{\prime}$. Let us fix $k$. Since $g_{k}$ is only a rescaling of $f, g_{k}$ is sense preserving and by Proposition 4.1 we have

$$
£\left(g_{k} \circ \mu, g_{k} \circ \nu\right)=1 .
$$

Through the homotopy

$$
H(\xi, \eta, t)=\left(g_{k} \circ \Phi\right)\left(\xi, t \eta_{0}\right)-\left(g_{k} \circ \Phi\right)\left(\mathbf{e}_{1}+t \xi_{0},-\eta\right) .
$$

we obtain that

$$
£\left(g_{k} \circ \varphi, g_{k} \circ \psi\right)=£\left(g_{k} \circ \mu, g_{k} \circ \nu\right)=1
$$

since $H(\xi, \eta, t) \neq 0$ for every $\xi \in \mathbb{S}_{1}, \eta \in \mathbb{S}_{1}$ and $t \in[0,1]$. The last can be easily seen from $t \eta_{0} \neq-\eta$ because $g_{k} \circ \Phi$ is a one-one mapping.

Now, by continuity of the degree, we pass to the limit and obtain

$$
£(g \circ \mu, g \circ \nu)=£(g \circ \varphi, g \circ \psi)=1 .
$$


(The first equality is easily seen by a homotopic argument as above.) Using Proposition 4.1 "backwards" we infer that $g$ is sense preserving, so that $J_{f}\left(x_{0}\right)>0$.

\section{The General CASE}

For the treatment of the general case, we need to prepare some material concerning the Lorentz spaces.

Lorentz spaces. If $f: \Omega \rightarrow \mathbb{R}$ is a measurable function, we define its distribution function $m(\cdot, f)$ by

$$
m(\sigma, f)=|\{x:|f(x)|>\sigma\}|, \quad \sigma>0,
$$

and the nonincreasing rearrangement $f^{\star}$ of $f$ by

$$
f^{\star}(t)=\inf \{\sigma: m(\sigma, f) \leq t\} .
$$

The Lorentz space $L^{p, 1}(\Omega)$ is defined as the class of all measurable functions $f: \Omega \rightarrow \mathbb{R}$ for which

$$
\int_{0}^{\infty} t^{\frac{1}{p}-1} f^{\star}(t) d t<\infty
$$

see e.g. [16].

The role of this Lorentz space consists in the fact that the set of functions such that $|D f| \in L^{p, 1}$ is continuously embedded into the space of continuous functions when $p$ is the dimension of the domain.

Let $\phi$ be a bounded positive nonincreasing function on $(0,+\infty)$ such that

$$
\int_{0}^{\infty} \phi(s)^{1 / p} d s<\infty
$$

We denote

$$
F_{\phi}(s)= \begin{cases}s \phi(s)^{\frac{1}{p}-1}, & s>0, \\ 0, & s=0 .\end{cases}
$$

and notice that $F_{\phi}$ is continuous and nondecreasing at $0+$. We denote by $\mathcal{F}_{p}$ the class of all Orlicz functions of form $F_{\phi}$ with $\phi$ satisfying (6.1).

Proposition 6.1. Suppose that $|\Omega|<+\infty$. Let $g \in L_{p, 1}(\Omega)$. Then there exists $F \in \mathcal{F}_{p}$ such that

$$
\int_{\Omega} F(g(x)) d x<\infty .
$$

Proof. See [9], Prop. 2.1. The boundedness of $\phi$ with $F=F_{\phi}$ is due to the fact that the function $\phi$ is obtained as a slight modification of the distribution function of $g$, so that $\sup \phi \leq|\Omega|$.

Proposition 6.2. Let $F \in \mathcal{F}_{p}$. Let $u \in W^{1,1}\left(\mathbb{S}_{p}\right)$ be a continuous function. Then

$$
\left(\operatorname{osc}_{\mathbb{S}_{p}} u\right)^{p} \leq C \int_{\mathbb{S}_{p}} F(|\nabla u|) d x
$$

where $C$ depends only on $p$ and $F$. Here $\operatorname{osc}_{\mathbb{S}_{p}} u=\operatorname{diam}\left(u\left(\mathbb{S}_{p}\right)\right)$ as usual.

Proof. This follows immediately from the "flat version" in [9], Thm. 3.2. 
Proof of Theorem 1.2. For $n=2,3$, the corresponding Lorentz space is just $L^{1}$ and this result is contained in Theorem 1.1. Also for $n>3$, the proof is analogous to the case $n=3$ and therefore we only sketch it and point out the differences. Recall that $p=[n / 2]$. If $n$ is even, we set $q=p-1$, otherwise $q=p$. We may assume that $|\Omega|<+\infty$. Since $L_{p, 1} \subset L_{q, 1}$ on domains of finite measure, by Proposition 6.1 there exist $F_{p} \in \mathcal{F}_{p}$ and $F_{q} \in \mathcal{F}_{q}$ such that such that $\int_{\Omega} F(|\nabla u|)<\infty$ with $F=F_{p}+F_{q}$.

Again we fix a point $x_{0}$ such that $J_{f}\left(x_{0}\right) \neq 0,(2.1)$ holds for $x_{0}$, and such that $x_{0}$ is a Lebesgue point for the functions $f, \nabla f$ and $F(|\nabla f|)$. If we define $g_{k}$ by (5.1), we observe similarly to (5.2) that

$$
F\left(\left|\nabla g_{k}\right|\right) \rightarrow F(|\nabla g|) \quad \text { in } L^{1}(B)
$$

since $x_{0}$ is a Lebesgue point of $F(|\nabla f|)$. Using (5.2) and (5.3) we can choose $r_{k}$ such that

$$
\sum_{k}\left\|g_{k}-g\right\|_{1,1}<\infty \quad \sum_{k}\left\|F\left(\left|\nabla g_{k}\right|\right)-F(|\nabla g|)\right\|_{1}<\infty .
$$

Then the function

$$
\theta(x)=F(|\nabla g(x)|)+\sum_{k}\left|F\left(\left|\nabla g_{k}(x)\right|\right)-F(|\nabla g(x)|)\right|, \quad x \in B
$$

is an integrable majorant to the sequence $\left\{F\left(\left|\nabla g_{k}\right|\right)\right\}$. Given $\eta \in \mathbb{B}_{p+1}\left(\frac{1}{3}\right)$, we denote

$$
\varphi_{\eta}(\xi)=\Phi(\xi, \eta), \quad \xi \in \mathbb{S}_{p}
$$

Similarly, given $\xi \in \mathbb{B}_{q+1}\left(\frac{1}{3}\right)$, we denote

$$
\psi_{\xi}(\eta)=\Phi\left(\mathbf{e}_{1}+\xi, \eta\right), \quad \eta \in \mathbb{S}_{q} .
$$

We say that $T=\varphi_{\eta}\left(\mathbb{S}_{p}\right)$ is a good sphere for a function $h \in W^{1,1}(B) \cap C(B)$ if $\left.h\right|_{T} \in W^{1,1}(T)$ and the derivatives of $\left.h\right|_{T}$ coincide a.e. with the corresponding directional derivatives of $h$.

Again using Fubini theorem we can fix $\eta_{0}$ and $\xi_{0}$ such that $T_{\varphi}:=\varphi_{\eta_{0}}\left(\mathbb{S}_{p}\right)$ and $T_{\psi}:=\psi_{\xi_{0}}\left(\mathbb{S}_{q}\right)$ are good spheres for each $g_{k}$, that $g_{k} \rightarrow g$ in $W^{1,1}\left(T_{\varphi}\right)$ and $W^{1,1}\left(T_{\psi}\right)$ and $F\left(\left|\nabla g_{k}\right|\right) \rightarrow F(|\nabla g|)$ in $L^{1}\left(T_{\varphi}\right)$ and $L^{1}\left(T_{\psi}\right)$ and $\theta \in L^{1}\left(T_{\psi}\right)$ and $L^{1}\left(T_{\varphi}\right)$. From monotonicity of $F$ we infer that

$$
F\left(\frac{1}{2}\left|\nabla g_{k}-\nabla g\right|\right) \leq F\left(\left|\nabla g_{k}\right|\right)+F(|\nabla g|) \leq 2 \theta .
$$

Passing to a subsequence we obtain that $\nabla g_{k} \rightarrow \nabla g$ a.e. on $T_{\varphi}$ and $T_{\psi}$. Since $F$ is continuous at $0+$, we obtain

$$
F\left(\frac{1}{2}\left|\nabla g_{k}-\nabla g\right|\right) \stackrel{k \rightarrow \infty}{\rightarrow} 0 \quad \text { a.e. on } T_{\varphi} \text { and } T_{\psi} .
$$

Using the Lebesgue dominated convergence theorem we obtain

$$
\begin{aligned}
\int_{T_{\varphi}} F\left(\frac{1}{2}\left|\nabla g_{k}-\nabla g\right|\right) d \mathcal{H}^{p} & \rightarrow 0, \\
\int_{T_{\psi}} F\left(\frac{1}{2}\left|\nabla g_{k}-\nabla g\right|\right) d \mathcal{H}^{q} & \rightarrow 0 .
\end{aligned}
$$

The oscillation estimate from Proposition 6.2 implies that $\operatorname{osc}_{M}\left(g_{k}-g\right) \rightarrow 0$, where $M=T_{\varphi} \cup T_{\psi}$. Since $g_{k} \rightarrow g$ in $L^{1}\left(T_{\varphi}\right)$ and $L^{1}\left(T_{\psi}\right)$, it follows that 
$g_{k} \rightarrow g$ uniformly on $M$. The same homotopy argument as in (5.4) gives us that $J_{f}\left(x_{0}\right)>0$.

\section{REFERENCES}

[1] K. Astala, T. Iwaniec and G. Martin, Elliptic partial differential equations and quasiconformal mappings in the plane, Princeton Mathematical Series, 48. Princeton University Press, Princeton, NJ, 2009.

[2] L. C. Evans and R. F. Gariepy, Measure theory and fine properties of functions, Studies in Advanced Mathematics, Boca Raton, 1992.

[3] I. Fonseca and W. Gangbo, Degree Theory in Analysis and Applications, Clarendon Press, Oxford, 1995.

[4] F. W. Gehring and O. Lehto, On the total differentiability of functions of a complex variable, Ann. Acad. Sci. Fenn. Ser. A I 272 (1959), 1-9.

[5] P. Hajłasz, private communication, 2002.

[6] S. Hencl, P. Koskela and J. Onninen, Homeomorphisms of bounded variation, Arch. Ration. Mech. Anal. 186 (2007), 351-360.

[7] T. Iwaniec and G. Martin, Geometric function theory and nonlinear analysis, Oxford Mathematical Monographs. The Clarendon Press, Oxford University Press, New York, 2001. .

[8] T. Iwaniec and G. Martin, Squeezing the Sierpiski sponge, Studia Math 149 (2002), $133-145$.

[9] J. Kauhanen, P. Koskela and J. Malý, On functions with derivatives in a Lorentz space, Manuscripta Math. 100 (1999), 87-101.

[10] J. Kauhanen, P. Koskela and J. Malý, Mappings of finite distortion: Condition N, Michigan Math. J. 49 (2001), 169-181.

[11] P. Koskela, Lectures on Quasiconformal Mappings, University of Jyväskylä, to appear.

[12] J. Malý, Examples of weak minimizers with continuous singularities, Exposition. Math. 13 no. 5 (1995), 446-454.

[13] T. Rado, P. V. Reichelderfer, Continuous Transformations in Analysis, Springer 1955.

[14] S. Rickman, Quasiregular mappings, Ergebnisse der Mathematik und ihrer Grenzgebiete (3) [Results in Mathematics and Related Areas (3)], 26. Springer-Verlag, Berlin, 1993.

[15] D. Rolfsen, Knots and links, Berkeley, 1976.

[16] E. M. Stein and G. Weiss, Introduction to Fourier Analysis on Euclidean Spaces, Princeton Univ. Press, 1971.

[17] D. Swanson and W. P. Ziemer, A topological aspect of Sobolev mappings, Calc. Var. Partial Differential Equations 14 no. 1 (2002), 69-84.

[18] W. K. Ziemer, A note on the support of a Sobolev function on a $k$-cell, Proc. Amer. Math. Soc. 132 no. 7 (2004), 1987-1995.

Department of Mathematical Analysis, Charles University, Sokolovská 83, 18600 Prague 8, Czech Republic

E-mail address: hencl@karlin.mff.cuni.cz

Department of Mathematical Analysis, Charles University, Sokolovská 83, 18600 Prague 8, Czech Republic, and

Department of Mathematics, J. E. Purkyně University, České mládeže 8, 40096 Ústí NAD LABEM, Czech Republic

E-mail address: maly@karlin.mff.cuni.cz 